\title{
Mothers' Knowledge, Attitude and Practice regarding Prevention of Diarrhea among Children: An Empirical Investigation \\ DOI: https://doi.org/10.47175/rissj.v2i4.326
}

\section{| Manjura Khatun ${ }^{1}$ | Afroza Sharmin ${ }^{2}$ | Ishrat Jahan Bithi ${ }^{3}$ |}

\author{
${ }^{1}$ Assistant Professor, \\ Department of Reproductive \\ and Child Health Enam Nursing \\ College, Savar, Dhaka \\ ${ }^{2}$ Assistant Professor, \\ Department of Hospital \\ Management Enam Nursing \\ College, Savar, Dhaka \\ ${ }^{3}$ Vice-Principal, Prince Nursing \\ College, Talbag, Thana Road, \\ Savar, Dhaka
}

\begin{abstract}
The descriptive type of cross-sectional study was carried out to find out the mothers' knowledge, attitude and practice regarding the prevention of diarrhea among children at Kamarpara Slum Area, Dhaka. In this study, 110 mothers were interviewed. It was found from the current study that the age distribution of the study population, out of total 110 respondents, $46.4 \%$ of the respondents belongs to the age group 20-29 years. The study showed that only $47.3 \%$ of women have completed primary education, majority of the respondents (69.1\%) lived in kacha house, above half of the respondents (62.7\%) agreed that diarrhea means passage of loose or watery stools $>3$ times in 24 hours, majority of the respondents (46.4\%) got information from TV, most of the respondent's children (88.2\%) suffered from diarrhea, most of the respondents (70.9\%) had given EBF to their child during diarrhea where as $29.1 \%$ didn't practice EBF during diarrhea. Most of the respondents (79.1\%) were aware about the causes of diarrhea, $74.5 \%$ respondents had knowledge on danger signs of diarrhea, 25.5\% didn't know about danger signs of diarrhea. $85.5 \%$ respondents said that diarrhea can be prevented by drinking clean water. $75.5 \%$ respondents said that hand washing prevents diarrhea, $46.4 \%$ respondents drinks water from Tube-well, majority of the respondents (55.5\%) used sanitary latrine, $36.4 \%$ used non-sanitary latrine, $20 \%$ respondents didn't wash their hands properly,33.6\% washed their hands with plain water, $58.2 \%$ respondents placing child's stool in toilet, $87.3 \%$ respondents gave ORS to their child during diarrhea, more than half of the respondents didn't know the preservation time of ORS, only $22.7 \%$ said the preservation time of ORSis $10-12$ hours. $81.8 \%$ of the respondents cut nail timely. In conclusion the knowledge of mothers on diarrhea was poor there is a need for extensive educational interventions of mothers about childhood diarrhea to reduce the suffering from dehydration of children.

KEYWORDS

knowledge; attitude; practice; diarrhea; mothers
\end{abstract}

\section{INTRODUCTION}

Diarrhea is the second major cause of child morbidity and death worldwide. Every year, there are 2.5 billion episodes and 1.5 million fatalities among children under five. This still accounts for $21 \%$ of all fatalities in poor nations. Diarrhea kills more children than AIDS, malaria, and measles combined. Moreover, it causes secondary infection. Diarrhea continues to kill over 3 million people yearly in the poor countries. (WHO) Diarrhea is the fifth greatest cause of mortality globally and continues to impact child health. In many poor countries, moms care for their children under five and treat most diarrheal episodes at home. They decide on the child's diet and the disease's overall treatment. So, itis vital they understand 
this prevalent illness. Individual and family efforts to prevent and/or manage diarrhea are critical to reducing diarrhea-related morbidity and mortality. (Shah et al, 2012, p. 627). Diarrhea is more common in developing countries than in developed countries. This is caused by several aspects, including the lack of adequate drinking water, lack of understanding of sanitation hygiene and poor nutritional status and public health status. It is estimated that 2.5 billion people have inadequate sanitation facilities and 1 billion people do not have access to clean water. (Lubis et al, 2021)

Home remedies are vital in the treatment of severe diarrhea. Because most societies have a cultural tradition of treating diarrhea patients at home or by themselves, this is the case. Diarrhea in children typically starts at home and continues after leaving the clinic. Studies indicate that caregivers notice diarrhea first, thus the choice to seek treatment is taken at home. Diarrhea is frequently treated at home using conventional and natural remedies. The doses of conventional therapies are often wrong and insufficient. This practice increases morbidity and mortality, as well as the risk of diarrheal illness. To avoid dehydration and nutritional harm, children must be treated at home for diarrhea. (Sijbesma et al, 2009, p.418).

Diarrhea is a significant health issue in poor nations. Diarrhea is still one of the leading causes of death in children under 5. Diarrhea causes $15-40 \%$ of all infant mortality in certain tropical nations. Every year, 1.8 billion people have diarrhea, and 3 million children under 5 dies from it. $80 \%$ of these fatalities are infants. Every year, gastroenteritis and diarrheal illnesses kill 110,000 children under the age of five in Bangladesh.

Diarrhea is the leading cause of mortality in Bangladesh. The high mortality rate is attributed to local belief and cultural practices, and mother's lack of understanding about diarrhea causation and prevention. Most diarrhea deaths in young infants may be avoided. This needless high mortality and morbidity related to diarrhea may be avoided by just educating moms. Diarrhea morbidity may be as high as one third of a child's first two years.

This study will provide baseline information about mothers' knowledge on diarrheas that the findings of the study will helps to understand the preventive measures and importance of correct management techniques of diarrhea. This will create awareness among the mothers. It also helps to reduce infant mortality and morbidity rate due to diarrheal diseases.

\section{RESEARCH METHODS}

Cross-sectional research was performed in Kamarpara slum, Dhaka. 110 moms agreed to take part in the research. Purposive non-probability sampling was used. A predesigned and pretested questionnaire was used to gather data on socio-demographic characteristics, risk factors for diarrhea, perceptions of diarrhea, knowledge, attitudes, and practices about prevention and treatment of diarrhea. The research subjects gave written informed consent. Participants' identities were protected, as was the data gathered. In order to avoid missing or inconsistent data, data were verified for completeness, accuracy, and internal consistency. The corrected data were analyzed using SPSS version 19. The study's goal guided the development of an analytical strategy. The research was approved by the institutional ethics committee.

Table 1. Distribution of the respondents by age $(n=110)$

\begin{tabular}{lcc}
\hline Age in years & Frequency & Percentage \\
\hline $15-19$ & 30 & 27.3 \\
$20-29$ & 51 & 46.4 \\
$30-39$ & 26 & 23.6 \\
$>40$ & 3 & 2.7 \\
\hline Total & 110 & 100.0 \\
\hline \multicolumn{3}{c}{ Mean \pm SD $=2.02 \pm .790$} \\
\hline
\end{tabular}


This table shows that $46.4 \%$ of the respondents belongs to the age group 20-29 years and other $27.3 \%$ were $15-19$ years. The mean age of the respondents was $2.02 \pm .790$.

Table 2. Distribution of the respondents by their occupation(n=110)

\begin{tabular}{lcc}
\hline Occupation & Frequency & Percentage \\
\hline Housewife & 75 & 68.2 \\
Service Holder & 22 & 20.0 \\
Business & 5 & 4.5 \\
Student & 2 & 1.8 \\
Other & 6 & 5.5 \\
\hline Total & 110 & 100.0 \\
\hline
\end{tabular}

This table revealed that the majority of respondents (68.2\%) were housewife. $20 \%$ were service holder, Business $4.5 \%$, Student $1.8 \%$ and others were $5.5 \%$.

Table 3. Distribution of the respondents by their educational status $(n=110)$

\begin{tabular}{lcc}
\hline Educational Status & Frequency & Percentage \\
\hline Illiterate & 38 & 34.5 \\
Primary & 52 & 47.3 \\
SSC & 14 & 12.7 \\
Bachelor & 1 & 9 \\
Master & 5 & 4.5 \\
\hline Total & 110 & 100.0 \\
\hline
\end{tabular}

Table no. 03 showed that $47.3 \%$ of women have completed primary education, $34.5 \%$ were illiterate, SSC $12.7 \%$, Bachelor .9\%, Master $4.5 \%$.
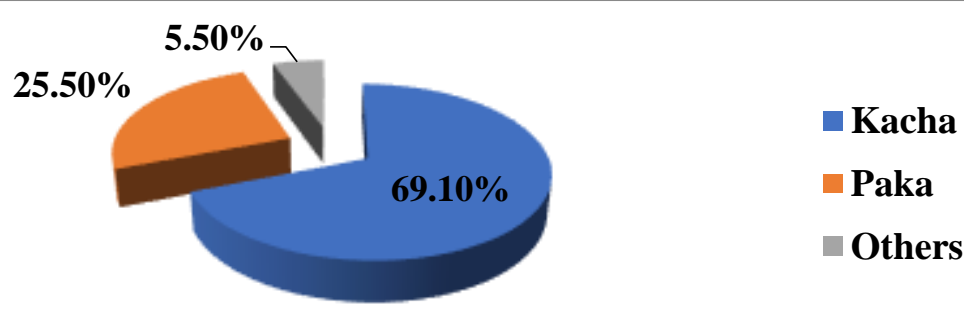

Figure 1. Distribution of the respondents by income $(\mathrm{n}=110)$

This figure reveals that most of the respondents $(69.1 \%)$ lived in kacha house, where rest of them $25.5 \%$ used to live in paka house and others $5.5 \%$.

Table 4. Distribution of the respondents by residence $(\mathrm{n}=110)$

\begin{tabular}{lcc}
\hline Monthly Income & Frequency & Percentage \\
\hline $2000-5000$ & 59 & 53.6 \\
$10000-14000$ & 40 & 36.4 \\
$15000-20000$ & 6 & 5.5 \\
$>20000$ & 5 & 4.5 \\
\hline Total & 110 & 100.0 \\
\hline
\end{tabular}

It is found from this table that $53.6 \%$ respondents had monthly income as 2000-5000 taka, $36.4 \%$ belongs to $10000-14000$ taka, $5.5 \%$ belongs to $15000-20000$ taka, $4.5 \%$ belongs to $>20000$ taka. 


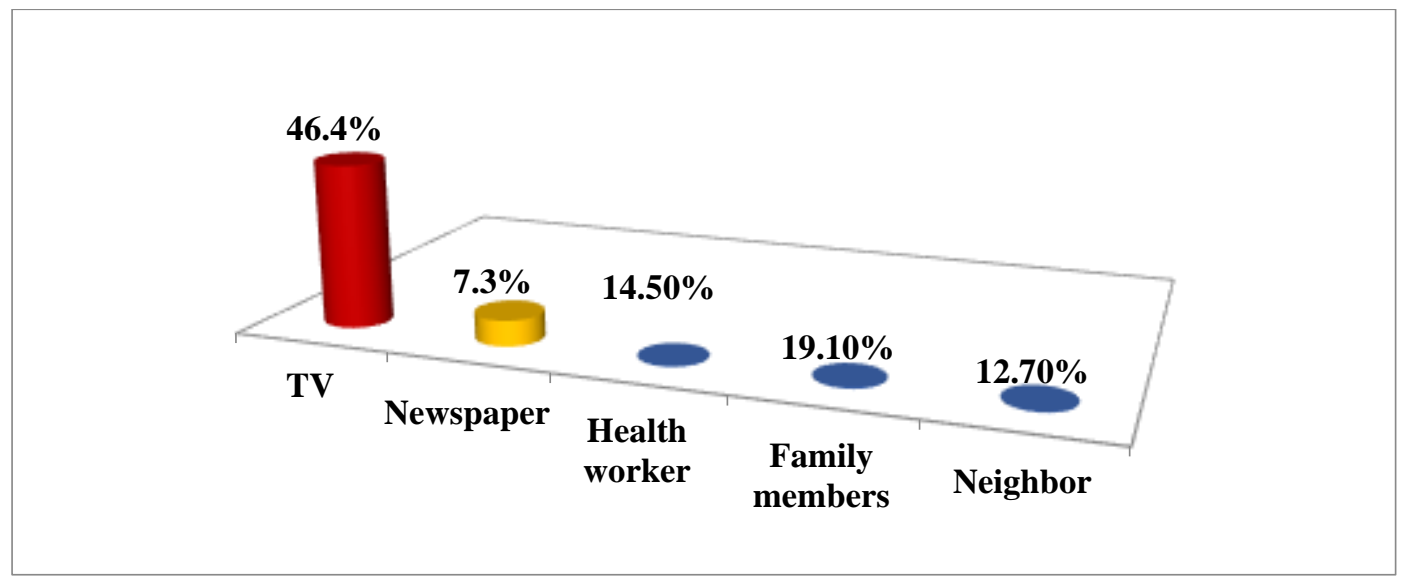

Figure 2. Distribution of the respondents by source of getting information $(n=110)$

This figure reveals that majority of the respondents $(46.4 \%)$ got information from TV,7.3\% Newspaper, 14.5\% Health Workers, 19.1\% Family Members, 12.7\% Neighbor.

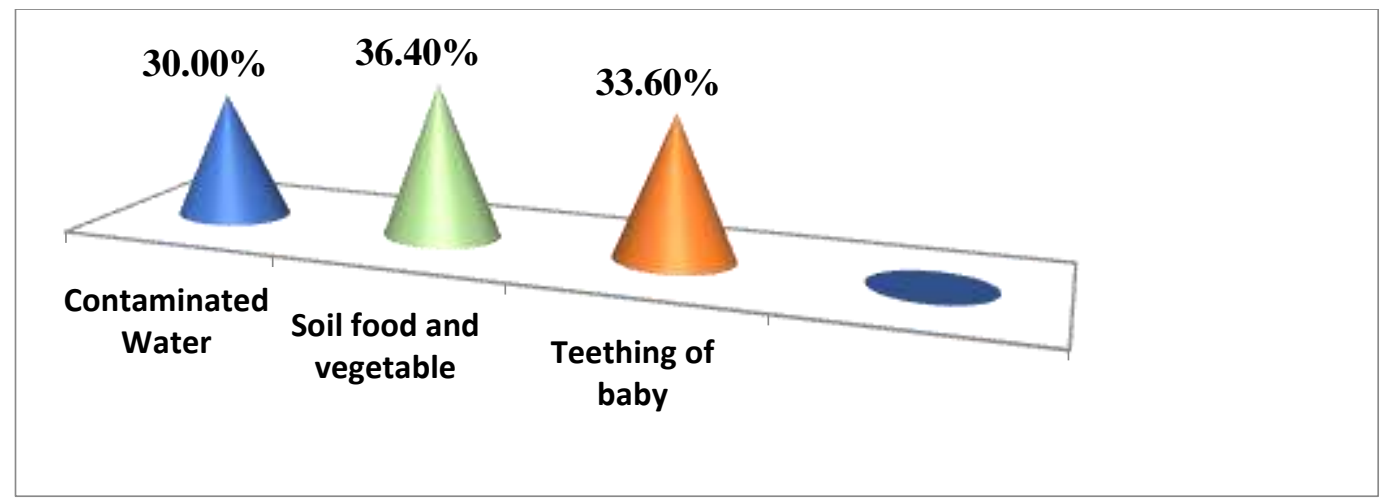

Figure 3. Respondent's awareness regarding causes of diarrhea $(n=110)$

This figure shows that $36.4 \%$ respondents agreed that diarrhea occurs due to soil food and vegetable, $33.6 \%$ said teething of baby, $30.0 \%$ respondents agreed that diarrhea occurs due to contaminated water.

Table 5. Distribution of the respondents by source of drinking water $(n=110)$

\begin{tabular}{lcc}
\hline Source of drinking water & Frequency & Percentage \\
\hline Boiled water & 20 & 18.2 \\
Filtered water & 14 & 12.7 \\
Tube-well water & 51 & 46.4 \\
Pond water & 4 & 3.6 \\
Supply water & 21 & 19.1 \\
\hline Total & 110 & 100.0 \\
\hline
\end{tabular}

This table reveals that $46.4 \%$ respondents' drinks water from tube-well, $19.1 \%$ supply water, $18.2 \%$ boiled water, $12.7 \%$ filtered water, and $3.6 \%$ pond water. 


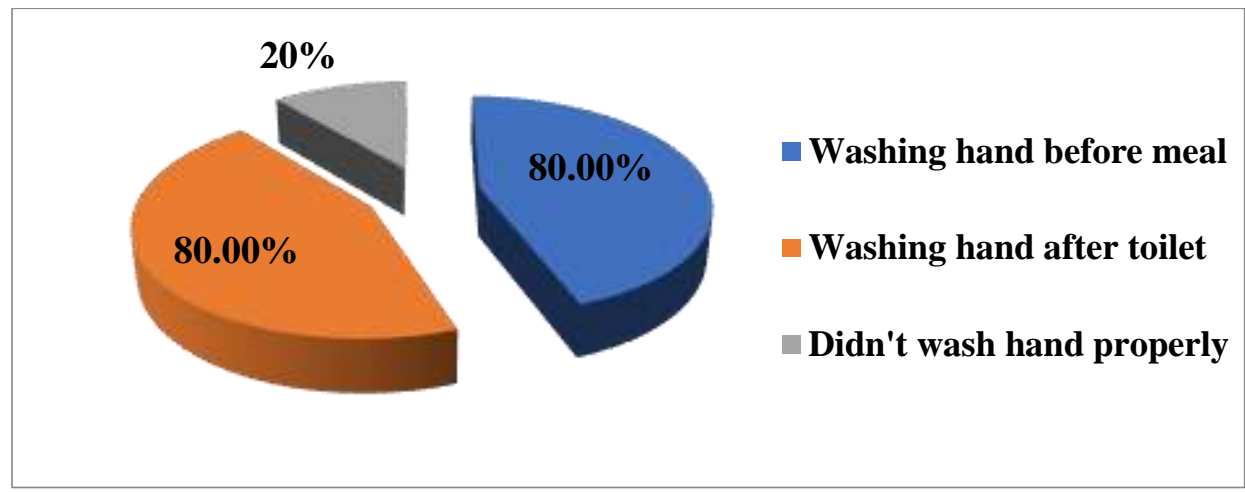

Figure 4. Distribution of the Respondent's awareness regarding hand washing $(n=110)$

This figure reveals that $80.0 \%$ respondents washed hand before meal and $80.0 \%$ respondents washed hand after toilet, where $20 \%$ respondents didn't wash their hands properly.

Table 6. Distribution of the respondents by knowledge on measures taken during diarrhea $(\mathrm{n}=110)$

\begin{tabular}{lcc}
\hline Measures taken during diarrhoea & Frequency & Percentage \\
\hline Homemade saline & 4 & 3.6 \\
Giving ORS & 96 & 87.3 \\
Collected medicine from shop without prescription & 10 & 9.1 \\
\hline Total & 110 & 100.0 \\
\hline
\end{tabular}

This table shows that $87.3 \%$ respondents gave ORS to their child during diarrhea, $9.1 \%$ respondents collected medicine from shop without prescription, $3.6 \%$ respondents gave homemade saline to their child during diarrhea.

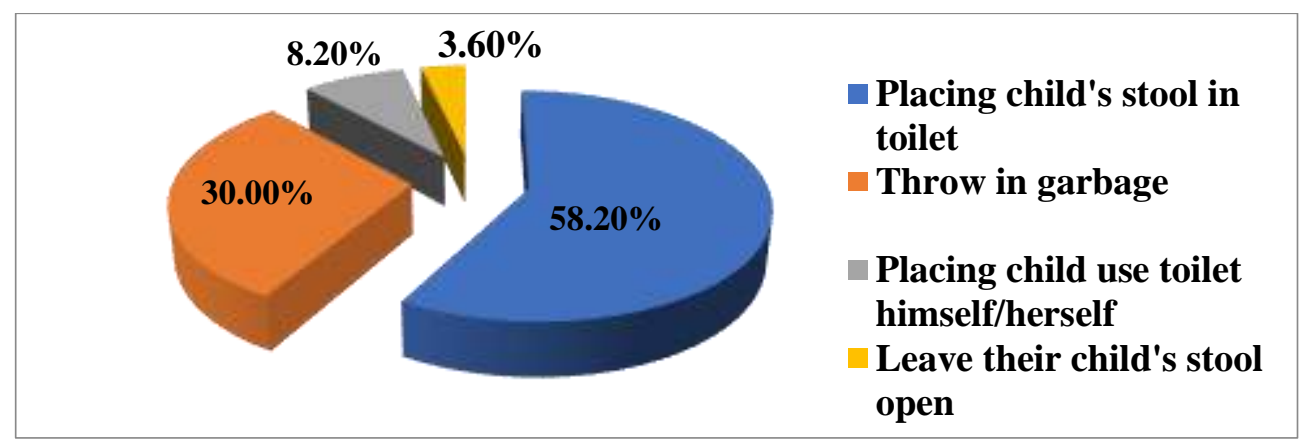

Figure 5. Respondent's knowledge on disposing child's stool $(\mathrm{n}=110)$

This figure shows that $58.2 \%$ respondents placing child's stool in toilet, $30 \%$ respondents throw in garbage, $8.2 \%$ respondent's child use toilet himself / herself, $3.6 \%$ respondents leave their child's stool open.

Table 7. Distribution of the respondents by knowledge on preservation time of ORS $(n=110)$

\begin{tabular}{lcc}
\hline Preservation time of ORS & Frequency & Percentage \\
\hline $10-12 \mathrm{hrs}$ & 25 & 22.7 \\
$>12 \mathrm{hrs}$ & 85 & 77.3 \\
Total & 110 & 100.0 \\
\hline
\end{tabular}

This table shows that more than half of the respondents $(77.3 \%)$ didn't know the preservation time of ORS, only $22.7 \%$ said the preservation time of ORS is $10-12 \mathrm{hrs}$. 
Table 8. Distribution of the respondents by knowledge on cutting nail timely $(n=110)$

\begin{tabular}{lcc}
\hline Cut nail timely & Frequency & Percentage \\
\hline Yes & 90 & 81.8 \\
No & 20 & 18.2 \\
Total & 110 & 100.0 \\
\hline
\end{tabular}

This table explore that $81.8 \%$ of the respondents their and their children's nail cut timely while only $18.2 \%$ of the respondents didn't cut nail timely.

\section{RESULTS AND DISCUSSION}

The study showed that only $47.3 \%$ of women have completed primary education, $34.5 \%$ were illiterate, SSC $12.7 \%$, bachelor. $9 \%$ and master $4.5 \%$. This study also showed that 78.2\% were Muslim, 20.9\% were Hindu and $0.9 \%$ were Christian. The current study reveals that most of the respondents $(69.1 \%)$ lived in kacha house, where rest of them $25.5 \%$ used to live in paka house and others $5.5 \%$. It is found that $53.6 \%$ respondents had monthly income as $2000-5000$ taka, $36.4 \%$ belongs to $10000-14000$ taka, $5.5 \%$ belongs to 15000 20000 taka, $4.5 \%$ belongs to $>20000$ taka.

It also reveals that majority of the respondents (46.4\%) got information from TV, $7.3 \%$ Newspaper, $14.5 \%$ Health Workers, $19.1 \%$ Family Members, $12.7 \%$ Neighbor. The present study shows that $88.2 \%$ respondents' children suffered from diarrhea and $11.8 \%$ respondents' children didn't suffer from diarrhea.

In this study shows that 34.5\% respondent's child suffered from diarrhea for 6 times, $24.5 \%$ respondent's child suffered from diarrhea for 3 times, $22.7 \%$ for 4 times, $10.9 \%$ for 8 times, $7.3 \%$ for 2 times. This table reveals that most of the respondents $(70.9 \%)$ had given EBF to their child during diarrhea where as $29.1 \%$ didn't practice EBF during diarrhea. This study shows that $36.4 \%$ respondents agreed that diarrhea occurs due to soil food and vegetable, $33.6 \%$ said teething of baby, $30 \%$ respondents agreed that diarrhea occurs due to contaminated water.

The current study shows that $74.5 \%$ respondents had knowledge on danger signs of diarrhea, $25.5 \%$ didn't know about danger signs of diarrhea. The study shows that $77.3 \%$ respondents agreed with thirst and dry mouth is a danger sign of diarrhea. It is observed from the current study shows that $85.5 \%$ respondents said that diarrhea can be prevented by drinking clean water. Other preventive measures known by the respondents includes washing hands before eating $83.6 \%$, using latrine for defecation $79.1 \%$, washing hands after passing stool $67.3 \%$, disposing children's faces into toilet $65.5 \%$, washing hands before feeding a child $52.7 \%$, washing hands after handling child's faces $43.6 \%$.

The study shows that $75.5 \%$ respondents said that hand washing prevents diarrhea, $72.7 \%$ said teething cause diarrhea, $68.2 \%$ said breast feeding should be continued during diarrhea, $61.8 \%$ said diarrhea attacks mostly bottle-fed children. It reveals that $46.4 \%$ respondents' drinks water from Tube-well. This study is dissimilar from the study that is conducted in Bangladesh on hygiene practices in rural areas where $95 \%$ of households obtain their drinking water from tube wells.

This study also reveals that majority of the respondents (55.5\%) used Sanitary latrine, $36.4 \%$ used non-sanitary latrine and $8.2 \%$ respondents defecate openly. The study reveals that $80.0 \%$ respondents washed hand before meal and $80.0 \%$ respondents washed hand after toilet, where $20 \%$ respondents didn't wash their hands properly. It is also reveals that almost $60.0 \%$ respondents washed hand with Soap, $33.6 \%$ with plain water and $6.4 \%$ with Ash.

The present study shows that $58.2 \%$ respondents placing child's stool in toilet, $30 \%$ respondents throw in garbage, $8.2 \%$ respondent's child use toilet himself / herself, $3.6 \%$ respondents leave their child's stool open. The study found shows that $87.3 \%$ respondents 
gave ORS to their child during diarrhea, $9.1 \%$ respondents collected medicine from shop without prescription, $3.6 \%$ respondents gave homemade saline to their child during diarrhea.

\section{CONCLUSION}

The research was conducted in Kkamarpara, Slumarea, Dhaka, Bangladesh. The results of this research revealed that mothers' understanding of causes of diarrhea, hand washing practices, drinking water, recognizing warning signals, prevention and treatment of diarrhea is lacking. Information, education, and communication efforts should be implemented to help mothers understand diarrhea management.

\section{Recommendation}

From the result, the writer has some recommendation such below:

1. Behavioral aspects in diarrhea management should be addressed as part of prevention efforts to reduce diarrheal illnesses.

2. Aims to improve awareness and reduce misconceptions among moms and the community as a whole via health education, community discussion, information distribution, and communication skills.

3. Encourage moms to wash their hands before feeding or urinating. Separate the cooking and food storage areas in the kitchen.

4. Not only government but also media can broadcast diarrhea related theme so that people can aware about diarrhea.

\section{REFERENCES}

Bhutta ZA. (2016). Acute Gastroenteritis in Children, Nelson textbook of pediatrics. Chapter 332.1323 .

Lubis, I., Indirawati, S. M., \& Marsaulina, I. (2021). The Coralation Between Sanitation Facilities and Personal Hygiene with the Cases of Diarrhea in Breastfeeding Toddlers in Sinabung Post-Eruption Settlements, Berastagi District, Karo Regency. Randwick International of Social Science Journal, 2(3), 241-249. https://doi.org/10.47175/rissj.v2i3.271

Pahwa S, Kumar GT, Toteja GS. (2010). "Population and Nutrition". Journal of Health. 28(6):553.

Ramachandran P, Gopalan HS. (2009). Under nutrition and risk of infections in preschool children. Nutrition Foundation of India, New Delhi, India. 130.

Rehan HS, Gautam K, Gurung K. (2003). "Mothers needs to know more regarding management of childhood acute diarrhea." Indian j. preventive social medicine. 34:1-2.

Shah D, Choudhury P, Gupta P, Mathew JL. (2012). Promoting Appropriate Management of Diarrhea: A Systematic Review of Literature for Advocacy and Action: UNICEF-PHFI Series on Newborn and Child Health, India. Indian Pediatrics. 49:627.

Sijbesma C, Christoffers T. (2009). "The value of hygiene promotion: cost effectiveness analysis of interventions in developing countries." Health Policy Planning. 24(6):418.

Sutaria S, Talsania N, Shah C. (2011). "Study of prevalence of diarrheal diseases among under five population." National Journal of Community Medicine. 2(1):96.

WaterAid. Hygiene. (2009). http://www.wateraid.org/uk/what_we_do/ the_need/5901.

WHO/UNICEF. (2004). Joint Statement Clinical Management of Acute Diarrhea. The United Nations Children's Fund/World Health Organization. 78:1192.

WHO. (2008). Weekly epidemiological record. 7.

World Health Report. (2006). Shaping the future, Rotavirus and Severe Childhood Diarrhea. world health organization. 12(2):304. 